\title{
Sciendo
}

\author{
BULGARIAN ACADEMY OF SCIENCES
}

CYBERNETICS AND INFORMATION TECHNOLOGIES • Volume 19, No 1

Sofia $2019 \quad$ Print ISSN: 1311-9702; Online ISSN: 1314-4081

DOI: $10.2478 /$ cait-2019-0009

\section{Adaptive Observer of Resistance in Sensorless Estimation of Speed and Position in Brushless DC Electric Motor}

\author{
Danail Slavov \\ Department Electrical Motion Automation Systems, Faculty of Automatics, Technical University of \\ Sofia, Kliiment Ohridski 8 Str., 1756 Sofia, Bulgaria \\ E-mail:d.slavov@tu-sofia.bg
}

\begin{abstract}
Estimating the speed and position using measurable electrical parameters would allow establishment of sensorless control systems for brushless DC motors, without the need to use expensive sensors for the rotor position and speed. When the motor is running, it heats up and the stator resistance rises. This heat-dependent change needs to be reflected in the observer, as it would produce an error in rated speed and position. An adaptive algorithm can compensate for the change of resistance as a disturbing effect of the motor heating. The adaptive algorithm for estimating the resistance is synthesized using the function of Lyapunov. This article is useful for estimation of brushless electric motor speed and position with observer. It contains simulations with an adaptive observer of resistance for sensorless estimation of speed and position in brushless DC motor through measurement of voltage and current.
\end{abstract}

Keywords: Brushless motor, observer, speed, position, estimate, Lyapunov, stator resistance.

\section{Introduction}

In the past years, the use of BrushLess DC motors (BLDC) in robotics has increased significantly. The establishment of various microcontroller systems and programming environments as well as their availability from different usage levels, combined with their low price, make them appropriate for control of the fast-acting IGBT and MOSFET modules [1,2]. This type of motors compared to other brush DC motors has the following advantages: Absence of brushes because they have permanent magnets on the rotor resulting in no losses there; high ratio of torque-tomoment of inertia; high power and small size; compact form; lack of sparks; higher torque at the same nominal power; smaller size compared to brush motors at the same power $[1,3]$. The control of this type of motors is realized based on they rotor position. This helps to avoid getting out of sync during impact loads and overloads of the motor shaft [4]. There are two ways of receiving information for the rotor position: the first one is through sensors (encoders and Hall sensors) and the other one utilizes sensorless acquisition of information by the means of an observer or 
estimators which can estimate the position based on the electrical quantities: current and voltage. There are various algorithms for position estimation (least squares method, Luenberger method, Kalman filter, etc.).

\section{Mathematical description of brushless DC electric motors}

By implementing the Kirchhoff's law for the electrical circuit of BLDC stator coil, a mathematical description of the motor can be obtained under the following assumptions: Eddy currents and hysteresis in the magnetic materials of the machine have insignificant impact on the phase currents; unsaturated motor; constant air gap; symmetric stator coils and constant active resistors and coil inductances; ideal power switches of the invertor $[3,14,15]$. These simplifications are completely acceptable for the aims of control and would not have significant influence on the accuracy of the motor's mathematical model. Equivalent circuit of the motor with a power part for stator coil control is shown in Fig. $1[3,14,16]$.

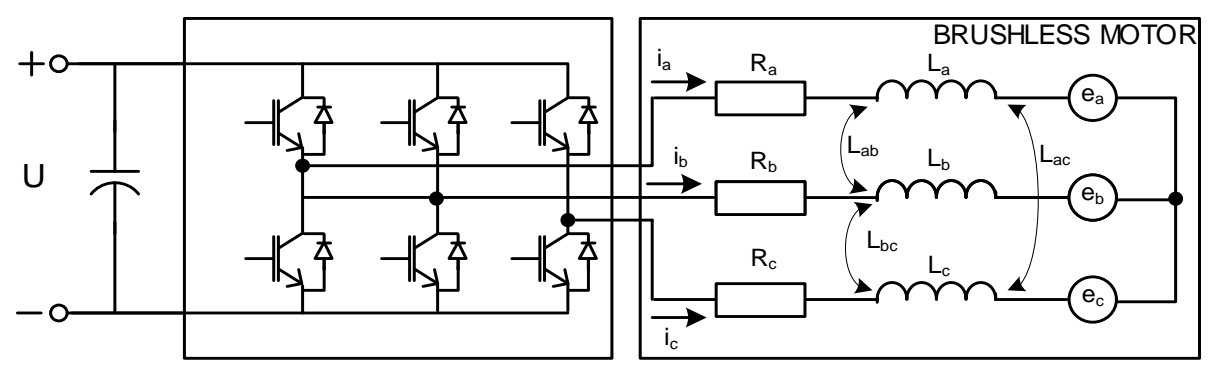

Fig. 1. BLDC with power part

Motor coils are connected in a wye configuration with isolated center, and for the phase currents, the following condition applies:

$$
i_{a}+i_{b}+i_{c}=0,
$$

$$
\left[\begin{array}{l}
u_{a} \\
u_{b} \\
u_{c}
\end{array}\right]=\left[\begin{array}{ccc}
R_{a} & 0 & 0 \\
0 & R_{b} & 0 \\
0 & 0 & R_{c}
\end{array}\right]\left[\begin{array}{l}
i_{a} \\
i_{b} \\
i_{c}
\end{array}\right]+\left[\begin{array}{ccc}
L_{a} & L_{a b} & L_{a c} \\
L_{b a} & L_{b} & L_{b c} \\
L_{c a} & L_{c b} & L_{c}
\end{array}\right]\left[\begin{array}{l}
i_{a} \\
i_{b} \\
i_{c}
\end{array}\right]+\left[\begin{array}{l}
e_{a} \\
e_{b} \\
e_{c}
\end{array}\right],
$$

where: $u_{a}, u_{b}, u_{c}$ are the phase voltages; $i_{a}, i_{b}, i_{c}$ - the phase currents; $e_{a}, e_{b}, e_{c}-$ the phase CEMFs; $R_{a}=R_{b}=R_{c}$ - the phase stator resistors; $L_{a}, L_{b}, L_{c}$ - the coils' own inductances; $L_{b a}, L_{c a}, L_{c b}, L_{a b}, L_{a c}, L_{b c}$ - the coils' mutual inductances.

The motor's phase counter-EMFs $[3,8,14]$ are determined by

$$
\left[\begin{array}{l}
e_{a} \\
e_{b} \\
e_{c}
\end{array}\right]=-\omega \frac{d}{d \theta}\left[\begin{array}{l}
\Phi_{a} \\
\Phi_{b} \\
\Phi_{c}
\end{array}\right]
$$

where: $\omega=d \theta / d t$ is the electric angular velocity of the rotor; $\theta$ - the electric rotation angle of the rotor; $\Phi_{a}, \Phi_{b}, \Phi_{c}$ - are magnetic fluxes for the stator phases $a, b$ and $c$. 
Having in mind that the three coils are equally symmetric and assuming that rotor magnetic resistance does not change, the following additional notations can be made $[3,9,14]$ :

$$
\begin{aligned}
& R_{a}=R_{b}=R_{c}=R_{s} ; L_{a}=L_{b}=L_{c}=L, \\
& L_{b a}=L_{c a}=L_{c b}=L_{a b}=L_{a c}=L_{b c}=L_{1} .
\end{aligned}
$$

Considering the above notations, Formula (1) can be presented in the following form:

$$
\left[\begin{array}{l}
u_{a} \\
u_{b} \\
u_{c}
\end{array}\right]=R_{s}\left[\begin{array}{l}
i_{a} \\
i_{b} \\
i_{c}
\end{array}\right]+L_{s} \frac{d}{d t}\left[\begin{array}{l}
i_{a} \\
i_{b} \\
i_{c}
\end{array}\right]+\left[\begin{array}{c}
e_{a} \\
e_{b} \\
e_{c}
\end{array}\right],
$$

where $L_{s}=L-L_{1}$ is the phase inductance.

3. Adaptive observer of the stator resistance for speed and position estimation

Mathematical model of BLDC is described in a stator $\alpha-\beta$ coordinate system by the following equations $[5,7]$ :

$$
\begin{array}{lr}
U_{\alpha}=R_{s} i_{\alpha}+L_{s} \frac{d i_{\alpha}}{d t}-\omega_{r} N_{p} \lambda_{r} \sin \theta_{r}, & \frac{d i_{\alpha}}{d t}=-\frac{R_{s}}{L_{s}} i_{\alpha}+\omega_{r} \frac{K_{e}}{L_{s}} \sin \theta_{r}+\frac{U_{\alpha}}{L_{s}} \\
U_{\beta}=R_{s} i_{\beta}+L_{s} \frac{d i_{\beta}}{d t}-\omega_{r} N_{p} \lambda_{r} \cos \theta_{r}, & \rightarrow \frac{d i_{\beta}}{d t}=-\frac{R_{s}}{L_{s}} i_{\beta}+\omega_{r} \frac{K_{e}}{L_{s}} \cos \theta_{r}+\frac{U_{\beta}}{L_{s}}
\end{array}
$$

$$
\frac{d \omega_{r}}{d t}=\frac{K_{T} N_{p}}{J}\left(i_{\beta} \cdot \cos \theta_{r}-i_{\alpha} \sin \theta_{r}\right)-\frac{B}{J} \omega_{r}, \quad \frac{d \theta_{r}}{d t}=\omega_{r},
$$

where $U_{\alpha}$ and $U_{\beta}$ are the voltages on $\alpha$ and $\beta$ axes respectively; $i_{\alpha}$ and $i_{\beta}$ are the corresponding currents on the same axes; $R_{s}$ is the stator resistance; $L_{s}$ is the stator inductance; $N_{p}$ is the number of motor poles; $\lambda_{r}$ is the flux; $K_{e}=N_{p} \lambda_{r}$ is coefficient of back emf; $K_{e}$ is torque constant; $\omega_{r}$ is the mechanical angular velocity; $\theta_{r}$ is the angular position of the rotor $[5,7]$.

The above four equations can be transformed into the state space

$$
\frac{d x}{d t}=A x+B u, \quad y=C x,
$$

where 
(9)

$$
\begin{aligned}
& x=\left[\begin{array}{c}
i_{\alpha} \\
i_{\beta} \\
\omega_{r} \\
\theta_{r}
\end{array}\right], u=\left[\begin{array}{c}
U_{\alpha} \\
U_{\beta}
\end{array}\right], y=\left[\begin{array}{c}
i_{\alpha} \\
i_{\beta}
\end{array}\right], B=\left[\begin{array}{cc}
\frac{1}{L_{s}} & 0 \\
0 & \frac{1}{L_{s}} \\
0 & 0 \\
0 & 0
\end{array}\right], C=\left[\begin{array}{llll}
1 & 0 & 0 & 0 \\
0 & 1 & 0 & 0
\end{array}\right], \\
& A=\left[\begin{array}{cccc}
-\frac{R_{s}}{L_{s}} & 0 & \frac{K_{e}}{L_{s}} \sin \theta_{r} & 0 \\
0 & -\frac{R_{s}}{L_{s}} & -\frac{K_{e}}{L_{s}} \cos \theta_{r} & 0 \\
-\frac{K_{T} N_{p}}{L_{s}} \sin \theta_{r} & \frac{K_{T} N_{p}}{L_{s}} \cos \theta_{r} & \frac{B}{J} & 0 \\
0 & 0 & 1 & 0
\end{array}\right] .
\end{aligned}
$$

The idea is to estimate $x(k)$ from the measurable $u(k)$ and $y(k)-$ Fig. 2. In this case, $u(k)$ represents the input voltage while the output $y(k)$ is the stator current.

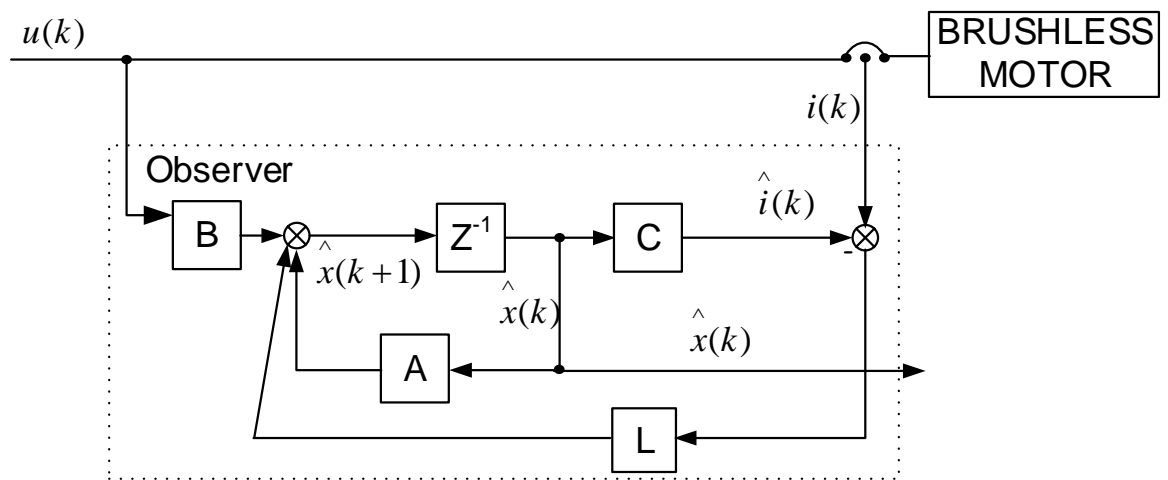

Fig. 2. Block-scheme of brushless motor observer

The effect of the temperature from the motor heating has an impact on the resistance value, which will grow with the temperature rise $[11,12]$. Therefore, when implementing brushless electric drives with observer, the parameters set herein should adequately reflect the real system parameters. Otherwise, each change in a parameter would lead to errors. The temperature impact on resistance can be compensated for by using an adaptive algorithm to assess it.

The equation of the estimated vector can be represented as follows [11]:

$$
\begin{gathered}
\frac{d}{d t} \hat{x}(t)=\hat{A} \hat{x}(t)+B u(t)+L(y(t)-C \hat{x}(t)), \\
\hat{i}_{a}=C \hat{x},
\end{gathered}
$$


where $C=\left[\begin{array}{lll}1 & 0 & 0\end{array}\right]$ - matrix of the output; $L$ - matrix in the observer feedback, chosen to ensure the necessary dynamics and stability.

With respect to resistance change due to coil heating during operation, the observer matrix represents a resistance function $\hat{A}=f\left(R_{a}\right)$. This requires the latter to be evaluated:

$$
\hat{A}=\left[\begin{array}{cccc}
-\frac{\hat{R}_{s}}{L_{s}} & 0 & \frac{K_{e}}{L_{s}} \sin \theta_{r} & 0 \\
0 & -\frac{\hat{R}_{s}}{L_{s}} & -\frac{K_{e}}{L_{s}} \cos \theta_{r} & 0 \\
-\frac{K_{T} p}{L_{s}} \sin \theta_{r} & \frac{K_{T} p}{L_{s}} \cos \theta_{r} & \frac{B}{J} & 0 \\
0 & 0 & 1 & 0
\end{array}\right] .
$$

Fig. 3 shows a block scheme of an observer with adaptive resistance assessment.

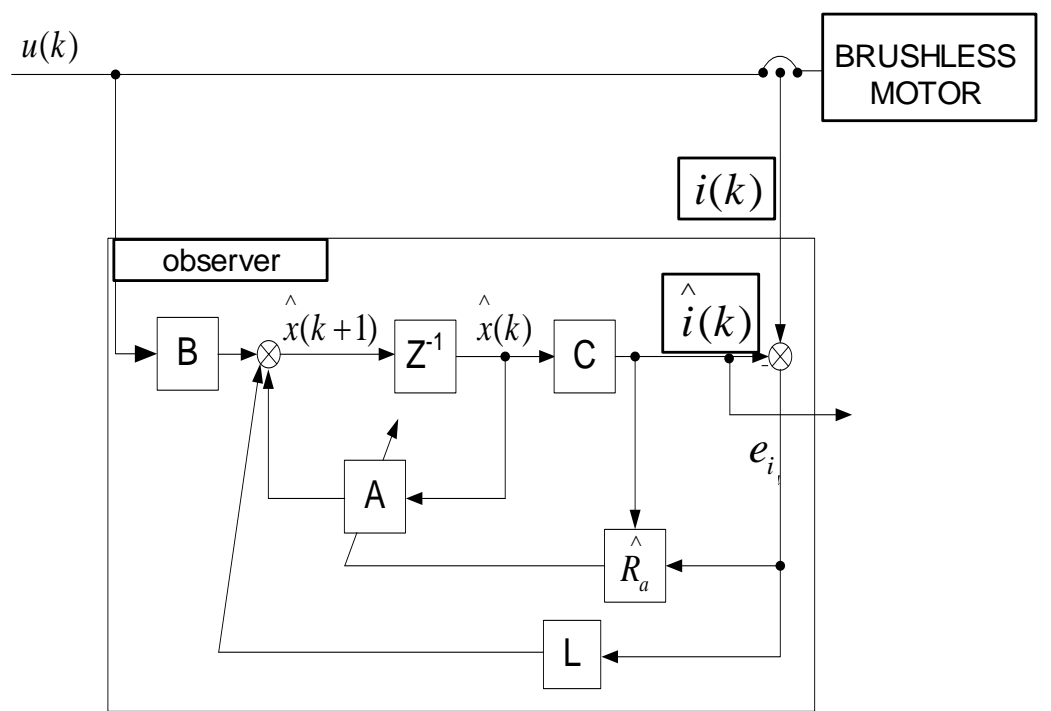

Fig. 3. Block scheme of observer with adaptive resistance estimation

For the synthesis of adaptive algorithm, Lyapunov theorem is applied to determine the error dynamics and stability in order to minimize the error to zero. [11].

The following applies to the calculation error

$$
e=x-\hat{x}
$$

or 


$$
\frac{d}{d t} e=\dot{x}-\hat{x}=(A x+B u)-[\hat{A} \hat{x}+B u+L(C x-C \hat{x})]
$$

(14)

where

$$
\begin{aligned}
& \frac{d}{d t} e=(A-L C)(x-\hat{x})+(A-\hat{A}) \hat{x}, \\
& \frac{d}{d t} e=(A-L C) e-\Delta A \hat{x},
\end{aligned}
$$

$$
\Delta A=\left[\begin{array}{lccc}
\frac{\hat{R_{s}}-R_{s}}{L_{s}} & 0 & 0 & 0 \\
0 & \frac{\hat{R_{s}}-R_{s}}{L_{s}} & 0 & 0 \\
0 & 0 & 0 & 0 \\
0 & 0 & 0 & 0
\end{array}\right] .
$$

Equation dynamics is determined by the proper values of $(A-L C)$. If these proper values have negative real parts, then the estimation of $\hat{x}$ approximates the actual $x[11,12]$.

The following Lyapunov function $V$ is chosen [11]:

$$
V=e^{\mathrm{T}} e+\frac{1}{2}\left(\hat{R}_{s}-R_{s}\right)^{2} \frac{1}{c},
$$

where $c$ is defined positive and satisfies the requirements of continuity and differentiability, $c>0=$ const.

The function $V$ equals 0 when both $(e)$ is zero and calculated resistance $\hat{R}_{a}$ equals the actual $R_{a}$ [11]. Sufficient condition for asymptotic resistance is derivative $d V / d t$ to be negative. This derivative is

$$
\begin{gathered}
\frac{d}{d t} V=e\left[\frac{d}{d t} e\right]^{\mathrm{T}}+e^{\mathrm{T}} \frac{d}{d t} e+\frac{1}{c} \frac{d \hat{R}_{s}}{d t}\left(\hat{R}_{s}-R_{s}\right)= \\
=e^{\mathrm{T}}\left[(A-L C)^{\mathrm{T}}+(A-L C)\right] e-2 \frac{\hat{R}_{s}-R_{s}}{L_{s}}\left(e_{i \alpha} \hat{i}_{s \alpha}+e_{i \beta} \hat{i}_{s \beta}\right)+2 \frac{d \hat{R}_{s}}{d x} \frac{\left(\hat{R}_{s}-R_{s}\right)}{c_{2}}, \\
\frac{d}{d t} V=e^{\mathrm{T}}\left[(A-L C)^{\mathrm{T}}+(A-L C)\right] e-e^{\mathrm{T}} \Delta A \hat{x}-e \Delta A^{\mathrm{T}} \hat{x}^{\mathrm{T}}+\frac{d \hat{R}_{s}}{d x}\left(\hat{R}_{s}-R_{s}\right),
\end{gathered}
$$

where $e_{i}=i-\hat{i}$ is the error between the measured and the estimated current. 
The condition of asymptotic stability is satisfied if $\frac{d}{d t} V<0$, i.e., $V$ decreases when $\left(\hat{R}_{s}-R_{s}\right)>0$. This is true if the sum of the last two terms is zero, which is the case when

$$
\frac{d \hat{R}_{s}}{d x}=\frac{c}{L_{s}}\left(e_{i \alpha} \hat{i}_{s \alpha}+e_{i \beta} \hat{i}_{s \beta}\right) .
$$

To ensure observer stability throughout the resistance range of variation, observer poles are chosen proportional to the motor poles. Proportionality constant is usually $k \geq 1$ which in turn makes the observer dynamically faster than the DC motor $[9,13]$. This is related to the sensitivity to noise (the larger this coefficient, the more sensitive the system is to noise). For real systems, a coefficient of proportionality up to 3 is recommended. Because the value of resistance depends on the current motor temperature, the matrix A should be calculated at each control step. This should be taken into account when implementing such systems, since they operate in real time and with a small sampling step. This requires significant response time and computation capabilities of the microcontroller in use.

The adaptive algorithm for evaluation of resistance based on Lyapunov theorem enables correction - in real-time during system operation - of the resistance value in the observer model [11]. This allows better operation of the electric drive system and its continuous adaptation to the varying working conditions.

In Matlab Simulink is generated s-function of level 2 for investigation of the observer with adaptive estimation of resistance. An $s$-function [13] is a block in Matlab where systems and algorithms for equation solving can be described by entering a special syntax. Its advantage is the ability for generating general-purpose blocks, which can then be used in various systems, changing their parameters accordingly.

\section{Simulation testing of an observer of BLDC electric drive speed and position with adaptive resistance}

Fig. 4 shows a Simulink model of the electric drive under study. A prebuilt control model for the BLDC motor speed is used from the demo folder of Simulink Brushless DC ac_example_simplifield with the following additions: Measurement block for voltage and current of the three phases, blocks for transformation of quantities from $a-b-c$ to stator $\alpha-\beta$ coordinate system; block for the observer synthesis implemented by a programming language which uses a level 2 s-function (OBSERVER).

The goal of this research is to compare estimated speeds and position with the actual quantities from the simulation model.

Simulation tests are accomplished in the Simulink environment of Matlab. The motor used for simulation has the following parameters: $T_{0}=25^{\circ} \mathrm{C}, L_{s}=0.0085 \mathrm{H}$, $l_{r}=0.175$ V.s $, J_{m}=0.089 \mathrm{~kg} . \mathrm{m}^{2}, B_{m}=1 \times e^{-3}(\mathrm{~N} . \mathrm{ms}) / \mathrm{rad}, R_{s}=2 \Omega, p=4$ poles. 
Simulations are conducted under the following conditions: Stator resistance value from catalog specifications at temperature $25^{\circ} \mathrm{C}, R_{s}=R_{s}\left(25^{\circ} \mathrm{C}\right)$, and resistance increase of $20 \%$ corresponding to 70 degrees Celsius.

At the simulation in time $t=0 \mathrm{~s}$, a step torque $T_{1}=3 \mathrm{~N}$.m is applied. Speed reference of $\omega_{y}=314 \mathrm{rad} / \mathrm{s}$ is applied step in $t=0 \mathrm{~s}$, and is stepwise reduced to $\omega_{y}=157 \mathrm{rad} / \mathrm{s}$ in $t=0.5 \mathrm{~s}$.

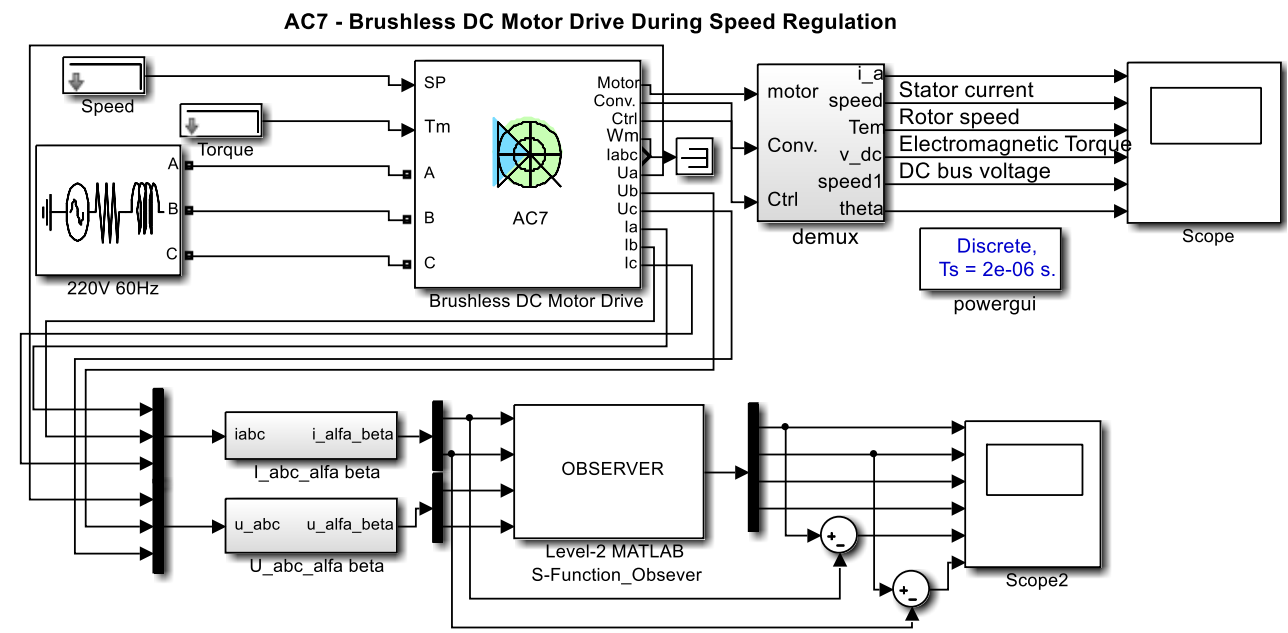

Fig. 4. Simulink model of an adaptive observer of resistance in sensorless estimation of speed and position in brushless DC electric motor

Figs 5-6 show the estimates, simulations, and errors between the estimated and simulated speeds and positions at $100 \% R_{s}=2 \Omega$.

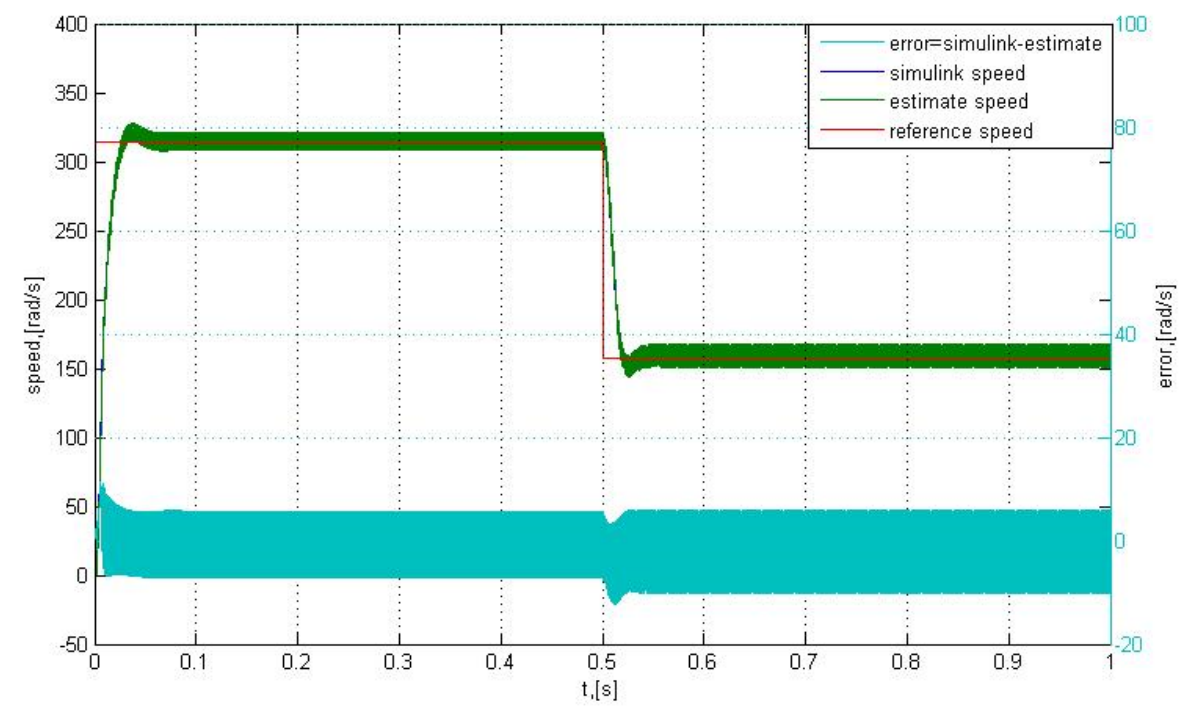

Fig. 5. Estimated and actual speed at $100 \% R_{s}=2 \Omega$ 


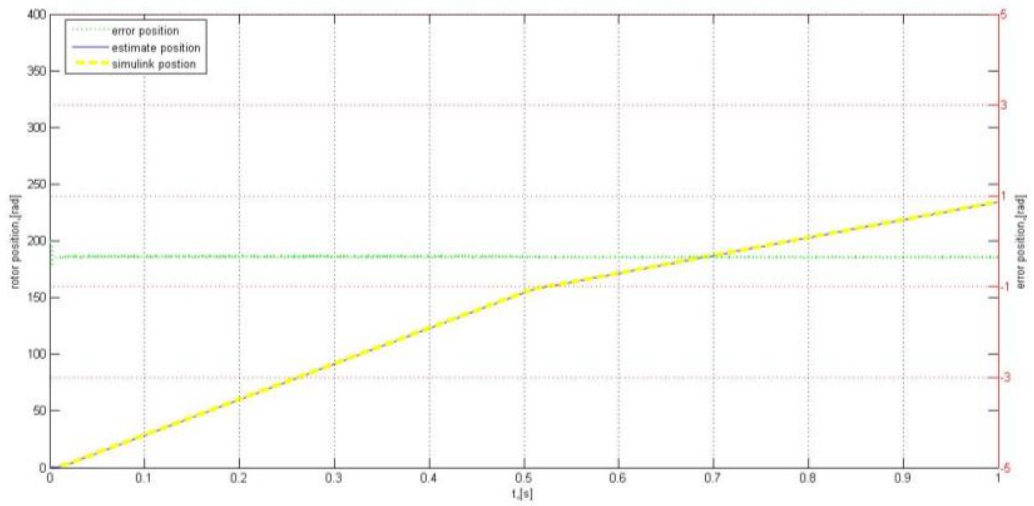

Fig. 6. Estimated and actual position at $100 \% R_{S}=2 \Omega$

Figs 7-8 show the estimates, simulations, and errors between the estimated and simulated speeds and positions at $120 \% R_{S}=2 \Omega$.

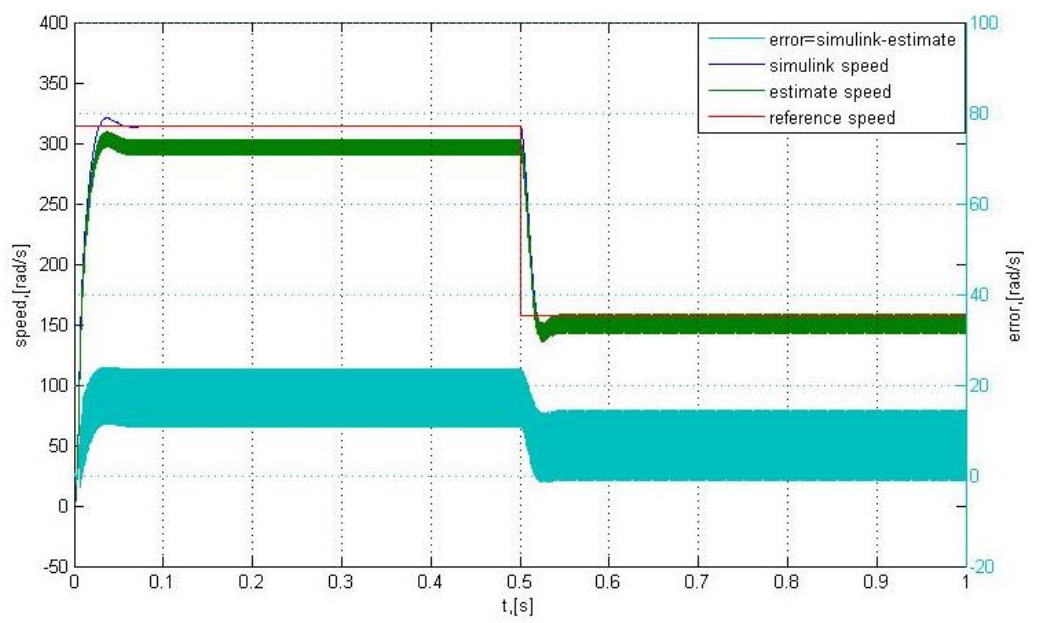

Fig. 7. Estimated and actual speed at $120 \% R_{S}=2 \Omega$

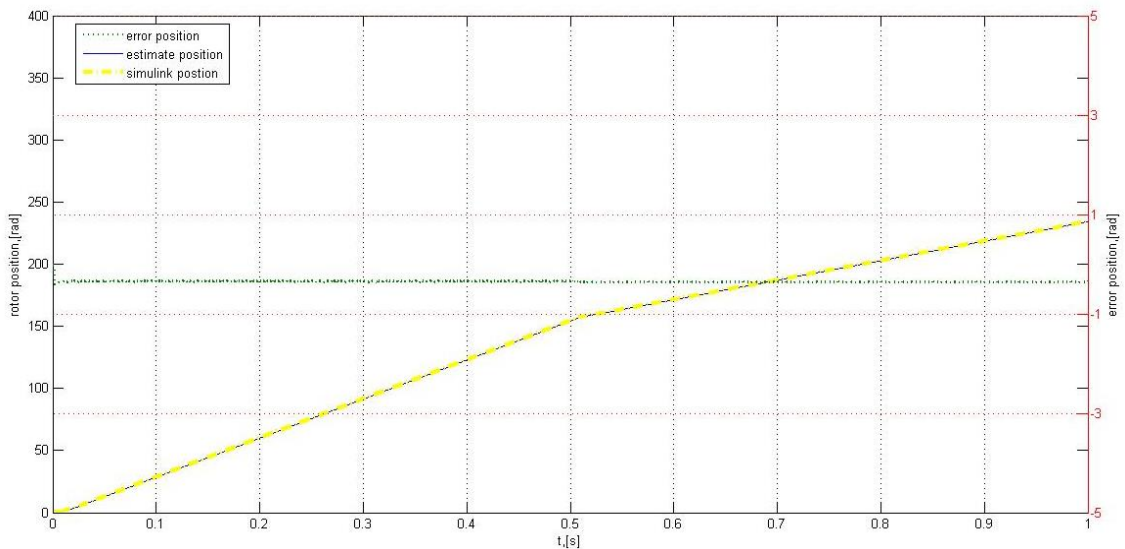

Fig. 8. Estimated and actual position at $120 \% R_{S}=2 \Omega$ 
Figs 9-10 show the estimates, simulations, and errors between the estimated and simulated speeds and positions at $120 \% R_{s}=2 \Omega$ and adaptive algorithm to estimate the stator resistance.

Summarized test results are shown in Table 1, with the following quality indicators chosen for the estimation [4,11]:

$e_{\omega s t e a d y}$ is the max error of the estimated speed in steady state,

$$
e_{\omega \text { steady }}=\max \left|\frac{\omega_{\text {sim }}-\hat{\omega}_{\text {estimate }}}{\omega_{\text {reference }}}\right| .100 \% ;
$$

$e_{\theta \max }$ is the max error of the estimated position over the tested period,

$$
e_{\theta \max }=\max \left|\frac{\theta_{\text {simulink }}-\hat{\theta}_{\text {estimate }}}{\theta_{\max }}\right| .100 \% .
$$

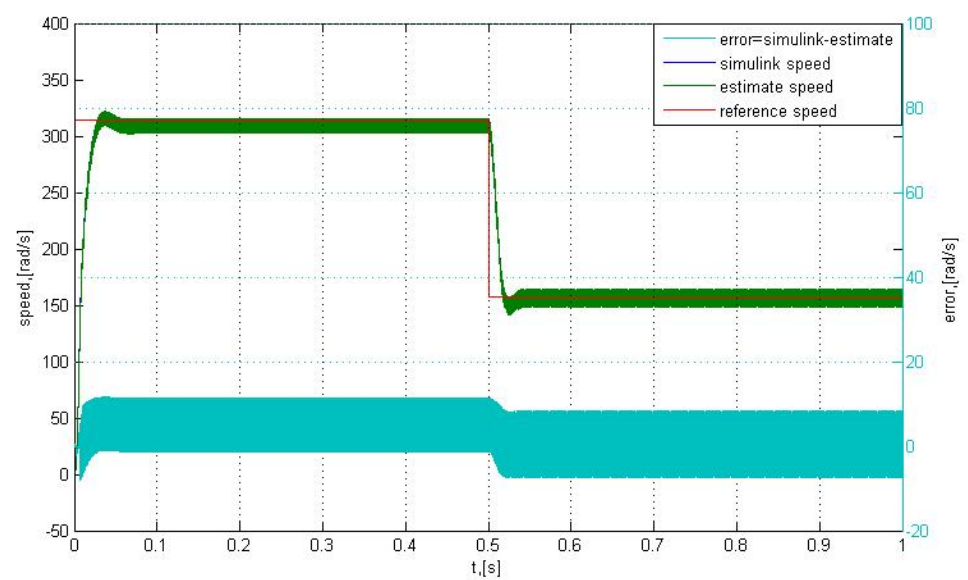

Fig. 9. Estimated and actual speed at $120 \% R_{S}=2 \Omega$

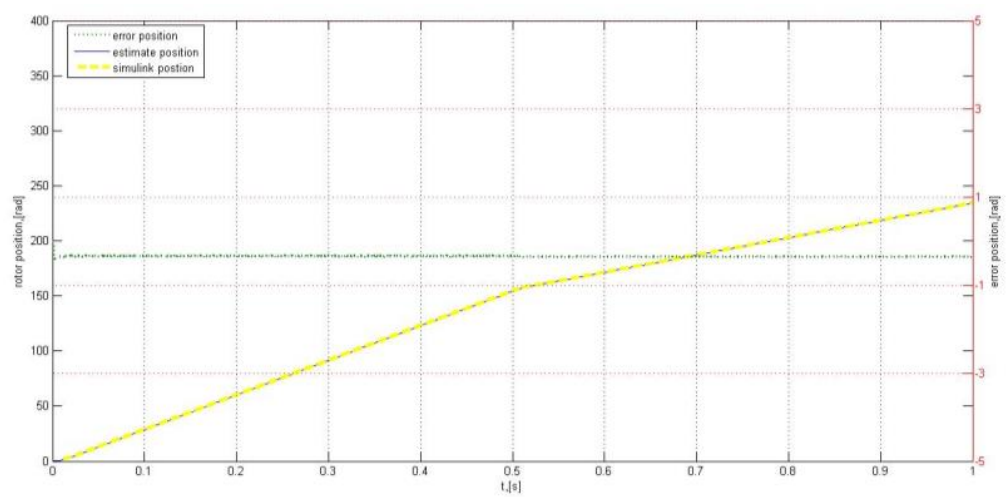

Fig. 10. Estimated and actual position at $120 \% R_{S}=2 \Omega$ 
Table 1. Summary of simulation results

\begin{tabular}{|c|c|c|c|c|c|}
\hline \multicolumn{2}{|c|}{$\begin{array}{c}\text { Observer at } \\
100 \% R_{S}=2 \Omega\end{array}$} & \multicolumn{2}{|c|}{$\begin{array}{c}\text { Observer at } \\
120 \% R_{S}=2 \Omega\end{array}$} & \multicolumn{2}{|c|}{$\begin{array}{c}\text { Observer with adaptive } \\
\qquad R_{S} \text { at } \\
120 \% R_{S}=2 \Omega \\
\end{array}$} \\
\hline $\begin{array}{c}e_{\max }, \\
\%\end{array}$ & $\begin{array}{c}e_{\text {costeady }} \\
\%\end{array}$ & $\begin{array}{c}e_{\theta \max } \\
\%\end{array}$ & $\begin{array}{c}e_{\text {asteady, }} \\
\%\end{array}$ & $\begin{array}{c}e_{\theta \max } \\
\%\end{array}$ & $\begin{array}{c}e_{\text {asteady }} \\
\%\end{array}$ \\
\hline 0.12 & 1.28 & 0.28 & 2.95 & 0.15 & 1.58 \\
\hline
\end{tabular}

\section{Conclusions}

The article provides a synthesis of observers for speed and position estimation by measuring the electrical quantities current and voltage in a brushless DC motor. These electrical quantities are easily measurable and suitable for speed and position estimation, and therefore can be used to make brushless systems which are cheaper than equivalent systems utilizing costly sensors (encoders and tachogenerators). In this article, observers are synthesized to assess the speed and position by measuring electrical current and voltage. The research is conducted by setting the motor parameters in the observer compliant with the catalog specification parameters at $25{ }^{\circ} \mathrm{C}$.

Continued brushless motor operation leads to an increase in these parameters due to heating. This is the cause for a resistance increase by $20 \%$, which corresponds to $70{ }^{\circ} \mathrm{C}$. Simulation is done to increase the impedance of brushless windings by $20 \%$, and the results show that the maximum error of the estimated speed in steady state marks a 2.3-fold increase compared to the absence of such resistance increase. The maximum error of the estimated position for the period of research also increases 2.3 times compared to the case without resistance change. This indicates a need for compensation implementation by means of coil resistance estimation, in order to reflect the temperature increase.

To achieve such resistance estimation and correct the observer's matrix, an algorithm synthesized by Lyapunov's function is proposed. In the synthesis of an observer which adapts according to the resistance (using Lyapunov's function), it is demonstrated that both maximum errors - of the estimated speed in steady state and of the estimated position - decrease by 1.9 and 2 times accordingly, compared to the situation without resistance adaptation.

The main conclusion is that when the motor temperature increases by $70{ }^{\circ} \mathrm{C}$, the use of an adaptive estimation to compensate for resistance provides significant reduction of the errors of estimated speed and position. To further reduce these errors and make them closer to the catalog values, a more accurate adjustment of the observer feedback coefficients and the Lyapunov's function coefficients is needed.

Simulation research shows that, depending on the required accuracy of the system, sensorless control systems for brushless DC motors are achievable. 


\section{References}

1. W a n g, S., C.-H. L u, A.-C. L e e. Disturbance Observer Structure Applied to SensorlessBrushless DC Motors Drive. - International Journal of Computer Theory and Engineering, Vol. 7, April 2015, No 2.

2. X i a, C.-L. Permanent Magnet Brushless DC Motor Drives and Controls. John Wiley \& Sons, Singapore, 2012. ISBN 978-1-118-18833-0.

3. Krishna n, R. Permanent Magnet Synchronous and Brushless DC Motor Drives. Taylor \& Francis, 2010. ISBN 978-0-8247-5384-9.

4. Hristo v, V. Influence of Change Armature Resistance on DC Motor when Use Observer. International Conference Automatics and Informatics, 2012, Journal 5-6/2012, pp. 101-104. ISSN 0861-7652.

5. U r b a n s ki, K. Sensorless Control of PMSM High Dynamic Drive at Low Speed Range. - In: 2011 IEEE International Symposium on Industrial Electronics (ISIE’11), 2011, pp. 728-732.

6. H r i s t o v, V. Adaptive Observer of DC Electric Drive. - Proceeding of Technical University Sofia, Vol. 63, 2013, Issue 2, pp. 89-98. ISSN1311-0829.

7. Kali appan, E., C. Che 11 a muthu. Modeling, Simulation and Experimental Analysis of Permanent Magnet Brushless DC Motors for Sensorless Operation. - Archives of Electrical Engineering, Vol. 61, 2012, No 4, pp. 499-515.

8. Li, H., S. Zh e n g, H. R e n. Self-Correction of Commutation Point for High-Speed Sensorless BLDC Motor with Low Inductance and Nonideal Back EMF. - IEEE Transactions on Power Electronics, Vol. 32, January 2017, No 1, pp. 642-651.

9. Moz aff ari Ni a p o u r, S. A. K. H., M. T a b a r r a i e, M. R. Fe y zi. A New Speed-Sensorless Robust Control Strategy for High-Performance Brushless DC Motor Drives with Reduced Torque Ripple. - Elsevier, Control Engineering Practice, Vol. 64, March 2014, pp. 42-54.

10. Mozaffari Niapour, S. A. K. H., M. Tabarrai e, M. R. Fe yzi. Design and Analysis of Speed-Sensorless Robust Stochastic L $\infty$-Induced Observer for High-Performance Brushless DC Motor Drives with Diminished Torque Ripple. - Elsevier, Energy Conversion and Management, Vol. 64, December 2012, pp. 482-98.

11. Hristov, V. Adaptive Algorithm for Estimating the Armature Resistance of DC Motor. Proceeding of Technical University Sofia, Vol. 62, 2012, Issue 4, pp. 69-78. ISSN1311-0829.

12. D a m o d h a r a n, P., K. V a s u d e v a n. Sensorless Brushless DC Motor Drive Based on the ZeroCrossing Detection of Back Electromotive Force (EMF) from the Line Voltage Difference. IEEE Transactions on Energy Conversion, Vol. 25, 2010, No 3, pp. 1-8.

13. L a i, Y.-S., Y.-K. L i n. Novel Back-EMF Detection Technique of Brushless DC Motor Drives for Wide Range Control Without Using Current and Position Sensors. - IEEE Transactions on Power Electronics, Vol. 23, 2010, No 2, pp. 934-940.

14. H r i s to v, V. Observer of Speed and Position Systems of Brushless DC Electric Motor. - In: International Annual Scientific Conference "Vasil Levski", 16-17 July 2015, pp. 214-224. ISSN 1314-1937.

15. C h e n g-H u, C. M in g, Y. C h e n g. A New Cost Effective Sensorless Commutation Method for Brushless DC Motors without Phase Shift Circuit and Neural Voltage. - IEEE Transactions on Power Electronics, Vol. 22, 2007, No 2, pp. 644-653.

16. Ni a pour, S. K. M., G. S. Garjan, M. Shafiei, M. R. Feyzi, S. Danyali, M. B. K o u h s h a h i. Review of Permanent-Magnet Brushless DC Motor Basic Drives Based on Analysis and Simulation Study. - International Review of Electrical Engineering (IREE), Vol. 9, 2014, No 5, pp. 930-957.

Received: 20.01.2019; Second Version: 27.01.2019; Accepted: 11.02.2019 (fast track) 